\title{
Autoionization of He atoms induced by partially stripped ion impact.
}

\author{
C.F. Martínez \\ Departamento de Física, Universidad Nacional del Sur, 8000 Bahía Blanca, Argentina. \\ S. Otranto* \\ Departamento de Física, Universidad Nacional del Sur, 8000 Bahía Blanca, Argentina. and \\ Consejo Nacional de Investigaciones Científicas y Técnicas (CONICET).
}

(Received on 25 January, 2010)

\begin{abstract}
A study of the autoionization process induced by partially stripped ion impact is performed. The BarrachinaMacek model is extended to the case in which the projectile-emitted electron interaction is represented by means of a non-Coulombic central potential at all times. Electron spectra in momentum space are predicted and compared to the Barrachina-Macek model and a recent model in which an asymptotically correct wave function was employed to treat this problem. The presence of rainbow scattering interference in the binary ring profile of the outgoing autoionized electrons for positive ion impact is discussed based on the different models.
\end{abstract}

Keywords: autoionization, post-collisional interactions, rainbow scattering

\section{INTRODUCTION}

One of the processes observed during the collision of charged particles and photons with atoms is the single ionization of the target which is left with one extra unit of positive charge. The ionization can be reached through direct single ionization or the two step Auger effect. For the latter, during the collision the incident particle (or photon) leaves the target atom in an excited state which then decays in a radiationless transition emitting an electron to the continuum. The term Auger effect is usually reserved for electron emission produced after an inner-shell vacancy is created in a manyelectron atom [1]. For the particular case in which a two electron atom is involved, the process is known as autoionization.

Autoionization can take place either induced by photon or ion impact. In the former, the emitted electron spectrum is isotropic provided that the emitted electron evolves only under the influence of the remaining target core. On the other hand, for the latter there is also the influence of the additional field of the projectile ion. Early studies on this issue were performed by Barker and Berry in 1966 who employed a classical model to describe the way in which the emitted electron spectrum profile departed from a Lorentzian [2]. For more than a decade, their model was used to describe autoionization following ion impact. In 1977, Devdariani et al. developed the first quantum mechanical model [3] that used the same assumptions as those of Barker and Berry, albeit in a quantal format. They assumed that the projectile distorted the electron's energy but not its trajectory. More recently, van der Straten and Morgenstern employed the Eikonal model in order to introduce the post collisional interaction (PCI) between the emitted electron and the projectile [4]. In 1989, Barrachina and Macek [5] successfully developed a continuum distorted wave (CDW) theory that predicted Coulomb focusing during the PCI between projectile and autoionized electron. Their predictions were experimentally verified the same year by Swenson et al. [6]. Thus, the energy and angular distribution of the ionized electrons are distorted by the presence of the projectile leading to a non-isotropic spectrum.

*Electronic address: sotranto@uns.edu.ar
This model with its subsequent improvements [7-9] account for the Stark effect (which is very important when the impinging ion energy is rather low) and the modification of the intermediate excited state lifetime due to the transient presence of the projectile.

Additional classical studies further concentrated in the classical description of the process trying to explain the Coulomb focusing mechanism in terms of a Coulomb path interference mechanism $[6,10]$. The authors concluded that this process is a consequence of the electrons being emitted in the forward direction at slightly different emission times so that the electrons follow different paths, but asymptotically have the same velocity. The Coulomb path interference thus represents an ion-atom collision analog to the classical double-slit electron mechanism.

These and other studies which concentrated on the nature of some oscillatory structures near the autoionization peak $[11,12,17]$ considered Coulomb-like fully stripped projectiles. However, it is known that partially stripped ions show more complex collision dynamics in single ionizing collisions. Nearly twenty years ago, oscillations in the emitted electron spectrum in the region of the binary peak have been experimentally observed and theoretically reproduced $[13,14]$. These oscillations have been attributed to interference structure in the elastic differential cross section for the scattering of target electrons from the impinging ion and have been associated with the well-known rainbow scattering [15].

In a recent work [16] Otranto and Olson considered the autoionization of $\mathrm{He}$ by partially stripped ion impact within the CDW model. In particular, they generalized the BarrachinaMacek model by replacing the Kummer function proper of the two body Coulomb problem in the continuum by an asymptotically correct wavefunction for this problem. This model, has the limitation of a doubtful applicability in the reaction zone (where the particles are still rather close to each other) as well as for small values of the relative velocity among the projectile and the emitted electron.

In this work, we concentrate on the autoionization of atoms by partially stripped ion impact. We develop a model in which a continuum wavefunction solution of a non-Coulombic central potential for the projectile-electron interaction is used to calculate the autoionization spectra. In spite of losing the analyticity of the previous models above cited, the present model 
provides a more accurate description of the collision system. In section II we point out the main features of the present model. Results are shown and discussed in section III. Finally, in section IV general conclusions are drawn.

Atomic units are used throughout this work unless explicitly stated otherwise.

\section{THEORETICAL QUANTUM MODELS}

We now describe the different distorted wave models that are employed in this work. The autoionization transition amplitude derived from the time-dependent perturbation theory can be expressed as [18],

$$
b(t)=-i \int_{0}^{t} d t^{\prime} e^{i\left(E-E_{0}\right) t^{\prime}-\frac{\Gamma}{2} t^{\prime}} t^{\prime i \frac{Z_{p}}{v_{P}}}\left\langle\Psi_{f}^{-}\left|\frac{1}{r_{12}}\right| \Phi_{i}\right\rangle
$$

where $\Psi_{f}^{-}$is the final state wave function, $\Phi_{i}$ the initial state wave function and $1 / r_{12}$ is the electronic repulsion between the two excited electrons.

\subsection{The Barrachina-Macek model}

In 1989 Barrachina and Macek proposed [5] the CDW approximation, which utilizes a distorted wave in order to represent the emitted electron-receding projectile interaction,

$$
\begin{aligned}
& \Psi_{f}^{-} \approx \Psi^{-}(\mathbf{r}) \Gamma\left(1+i Z_{p} / v^{\prime}\right) e^{\frac{\pi Z_{p}}{2 v^{\prime}}} \\
& \times{ }_{1} F_{1}\left[-i Z_{p} / v^{\prime} ; 1 ;-i\left(v^{\prime}\left|\mathbf{r}-\mathbf{v}_{P} t\right|+\mathbf{v}^{\prime} \cdot\left(\mathbf{r}-\mathbf{v}_{P} t\right)\right)\right]
\end{aligned}
$$

where $\mathbf{r}$ is the emitted electron-target radius. Since the integrand of the matrix element $\left\langle\Psi_{f}^{-}\left|\frac{1}{r_{12}}\right| \Phi_{i}\right\rangle$ is strongly confined to the target nucleus a peaking approximation can be performed leading to the simplified expression,

$$
\begin{aligned}
& b_{B M}=-i A_{0} \Gamma\left(1-i Z_{p} / v^{\prime}\right) e^{\frac{\pi Z_{p}}{2 v^{\prime}}} \\
& \times \int_{0}^{\infty} d t^{\prime} e^{i\left(E-E_{0}\right) t^{\prime}-\frac{\Gamma}{2} t^{\prime}} t^{\prime i \frac{Z_{p}}{v_{P}}}{ }_{1} F_{1}\left[i Z_{p} / v^{\prime} ; 1 ; i\left(v^{\prime} v_{P} t^{\prime}-\mathbf{v}^{\prime} \cdot \mathbf{v}_{P} t^{\prime}\right)\right]
\end{aligned}
$$

This expression separates the postcollisional interaction from a target dependent factor $A_{0}$ and leads to an analytical expression for the transition amplitude since the integral can be solved using standard techniques [19],

$$
\begin{aligned}
b_{B M}= & A_{0} \Gamma\left(1-i Z_{p} / v^{\prime}\right) \Gamma\left(1+i Z_{p} / v_{P}\right) e^{-\frac{\pi Z_{p}}{2 v_{P}}\left[1-\frac{v_{P}}{v^{\prime}}\right]} \\
& \times\left(E-E_{0}+i \Gamma / 2\right)^{-1-i Z_{p} / v_{P}} \\
& \times{ }_{2} F_{1}\left[i \frac{Z_{p}}{v^{\prime}} ; 1+i \frac{Z_{p}}{v_{P}} ; 1 ;-\frac{v_{P} v^{\prime}-\mathbf{v}_{P} \cdot \mathbf{v}^{\prime}}{E-E_{0}+i \Gamma / 2}\right]
\end{aligned}
$$

This separability allows a straightforward extension of the CDW model to more complex systems as has been shown in the analysis of the $N e K-L_{2,3} L_{2,3}\left({ }^{1} D_{2}\right)$ Auger line by Víkor et al. [20].

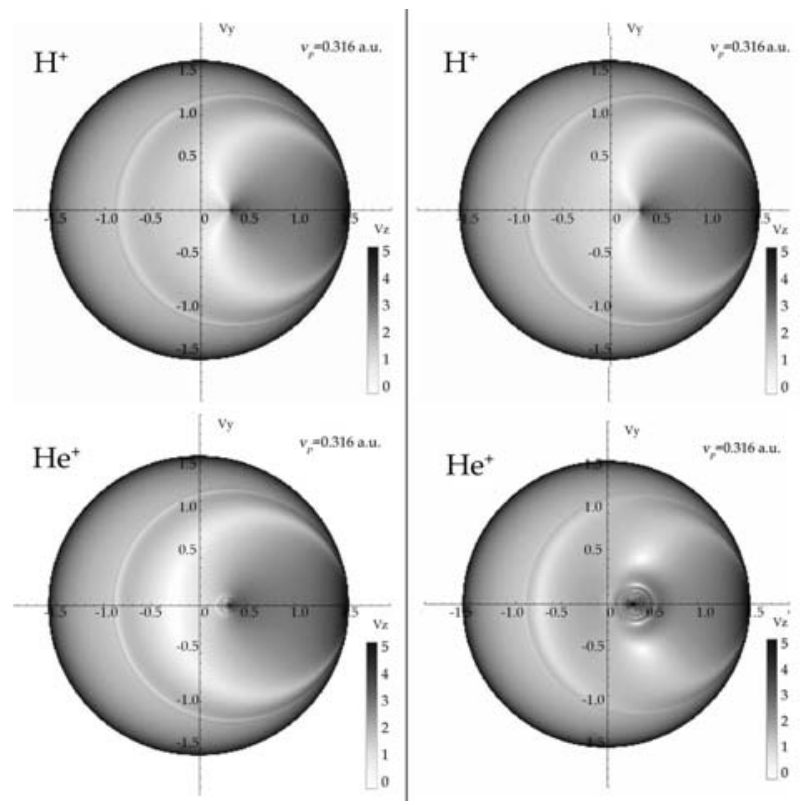

FIG. 1: Electron distribution in velocity space for the $2 s^{2}\left({ }^{1} S\right)$ autoionizing state of $\mathrm{He}$ by $\mathrm{H}^{+}$and $\mathrm{He}^{+}$projectiles. Left-column: $\mathrm{CP}-$ CDW model Right-column: ACP-CDW model. The impact velocity is 0.316 a.u.

\subsection{The ACP-CDW model}

For partially stripped ions however, a full solution requires the numerical evaluation of the radial equation for a central potential out to large distances since the integral converging factor $\Gamma / 2$ is target dependent and usually rather small. In order to give a qualitative description of the new features involved for clothed ions impact, the following approximation which is hereafter termed Asymptotic Clothed ProjectileCDW model (ACP-CDW) was introduced by Otranto and Olson in 2005. The main advantage is that it keeps the analyticity that is so useful in the CDW model,

$$
\begin{aligned}
& \left\langle\Psi_{f}^{-}\left|\frac{1}{r_{12}}\right| \Phi_{i}\right\rangle \approx A_{0}\left(\Gamma\left(1-i Z_{p} / v^{\prime}\right) e^{\frac{\pi Z_{p}}{2 v^{\prime}}}\right. \\
& \times{ }_{1} F_{1}\left[i Z_{p} / v^{\prime} ; 1 ; i\left(v^{\prime} v_{P}-\mathbf{v}^{\prime} \cdot \mathbf{v}_{P}\right) t\right] \\
& \left.+\frac{e^{i\left(v_{P} v^{\prime}-\mathbf{v}_{P} \cdot \mathbf{v}^{\prime}\right) t}}{v_{P} t} f_{n C}(\theta) e^{i \frac{Z_{p}}{v^{\prime}} \ln \left[2 v v^{\prime} t\right]}\right) .
\end{aligned}
$$

This wave function provides the correct asymptotic limit of the exact emitted electron-projectile wave function by including the non-Coulomb part of the scattering amplitude. The scattering amplitude obtained with this model for a nonCoulomb potential reads [21], 


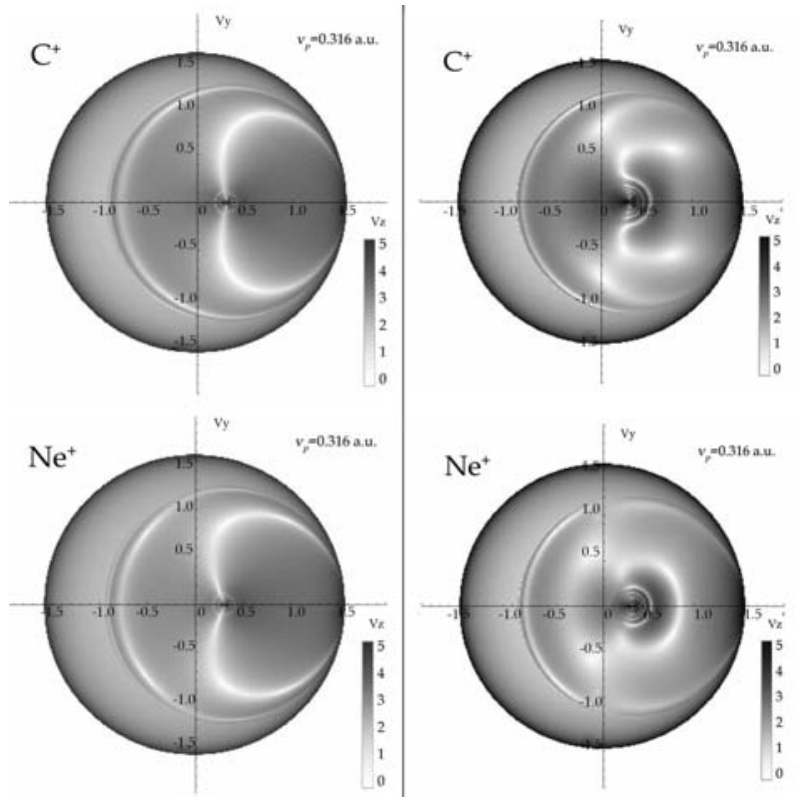

FIG. 2: Same as Figure 1 for $\mathrm{C}^{+}$and $\mathrm{Ne}^{+}$projectiles.

$$
\begin{aligned}
f_{m} & =f_{C}(\theta)+f_{n C}(\theta) \\
f_{n C}(\theta) & =\sum_{l=0}^{\infty} \frac{1}{v^{\prime}}(2 l+1) e^{i\left(2 \eta_{l}+\delta_{l}\right)} \sin \delta_{l} P_{l}(\cos \theta)
\end{aligned}
$$

where we denote by $v^{\prime}$ the electron-projectile relative velocity, $\eta_{l}=\arg \left(\Gamma\left(1+l-i Z_{p} / v^{\prime}\right)\right)$ is the Coulomb phase shift of the $l$ partial wave, $\delta_{l}$ is the non-Coulomb part phase shift and can be obtained by matching the logarithmic derivative of the inner and outward wave functions in the asymptotic region. The Coulomb scattering amplitude $f_{C}(\theta)$ is given by,

$$
f_{C}(\theta)=\frac{1}{i} \frac{\Gamma\left(1-i Z_{p} / v^{\prime}\right)}{\Gamma\left(i Z_{p} / v^{\prime}\right)} \frac{e^{i \frac{Z_{p}}{v^{\prime}} \ln \left[\frac{1-\cos \theta}{2}\right]}}{v^{\prime}(1-\cos \theta)}
$$

where $P_{l}(\cos \theta)$ represents the $l$ th order Legendre polynomial [22].

The ACP-CDW amplitude is then obtained after replacing Eq.(5) into Eq. (1) and yields,

$$
\begin{aligned}
b_{A C P-C D W}= & b_{B M}+A_{0} \frac{1}{v_{P}}\left(2 v^{\prime} v_{P}\right)^{i \frac{Z_{p}}{v^{\prime}}} \\
& {\left[\frac{\Gamma}{2}-i\left(E-E_{0}+v^{\prime} v_{P}-\mathbf{v}^{\prime} \cdot \mathbf{v}_{P}\right)\right]^{-i\left(\frac{Z_{p}}{v_{P}}+\frac{Z_{p}}{v^{\prime}}\right)}(8) } \\
& \times \Gamma\left(i\left(Z_{p} / v_{P}+Z_{p} / v^{\prime}\right)\right) f_{n C}(\theta) .
\end{aligned}
$$

For a partially stripped ion, the form of the potential is one which is parametrized in such a way that a large range of ionic

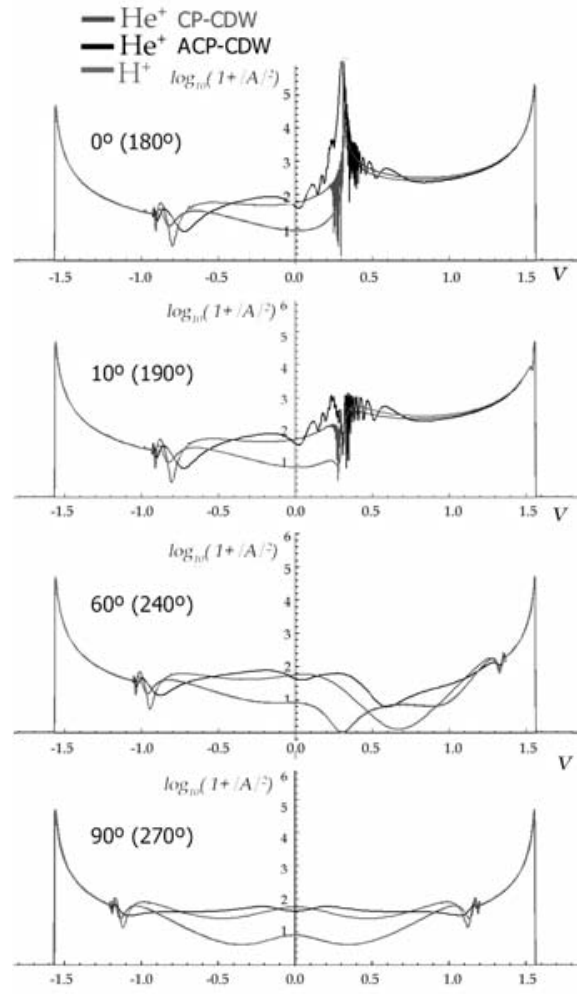

FIG. 3: $\mathrm{He}^{+}$impact velocity profile of $\log _{10}\left(1+|b|^{2}\right)$ for selected cuts of the velocity space: a) $\theta_{\text {emitter }}=0^{\circ}\left(180^{\circ}\right)$, b) $\theta_{\text {emitter }}=$ $10^{\circ}\left(190^{\circ}\right)$, c) $\theta_{\text {emitter }}=60^{\circ}\left(240^{\circ}\right)$, d) $\theta_{\text {emitter }}=90^{\circ}\left(270^{\circ}\right)$.

species may be easily considered. Thus, to model the emitted electron-projectile interaction we use the Hartree-Fock model potential of Garvey et al. [23] which simulates the interparticle separation dependent screening of the ion nuclear charge experienced by the electron and is given by,

$$
\begin{aligned}
V_{P}(r) & =\frac{[(N-1)(1-\Omega(r))-Z]}{r} \\
\Omega(r) & =\left[\left(\frac{\eta}{\xi}\right)\left(e^{\xi r}-1\right)+1\right]^{-1}
\end{aligned}
$$

Here, $(N-1)$ is the number of electrons present in the ion and $\eta$ and $\xi$ are the screening parameters which have been tabulated for ions $2 \leqslant Z \leqslant 54$. For heavier ions, the parameters may be found by a fit which utilizes points for a reduced parameter form of this potential [24].

The drawback of the model is its inaccurate description of the electrons which are emitted in the forward direction with velocities very close to that of the projectile.

\subsection{The CP-CDW model}

In addition to the limitations exhibited by the previous model around $v^{\prime} \approx 0$, we note that physical processes involving initial bound states highlight the role of the reaction region in which all the particles are rather close to each other. Thus, an asymptotic model does not guarantee a proper de- 


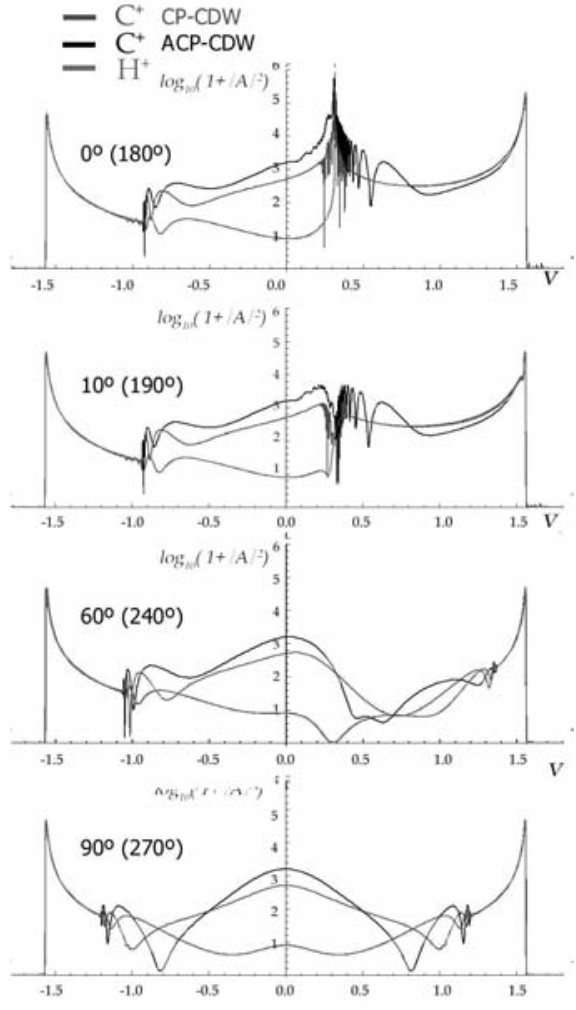

FIG. 4: $\mathrm{C}^{+}$impact velocity profile of $\log _{10}\left(1+|b|^{2}\right)$ for selected cuts of the velocity space: a) $\theta_{\text {emitter }}=0^{\circ}\left(180^{\circ}\right)$, b) $\theta_{\text {emitter }}=$ $10^{\circ}\left(190^{\circ}\right)$, c) $\theta_{\text {emitter }}=60^{\circ}\left(240^{\circ}\right)$, d) $\theta_{\text {emitter }}=90^{\circ}\left(270^{\circ}\right)$.

scription of the collision system in this region and the accuracy of the results obtained should be further examined.

In order to circumvent these issues we now propose a model based on a partial wave expansion for the emitted electron-projectile wave function. We hereafter term this model as Clothed Projectile CDW model or CP-CDW. In this way a more precise description of the electron-projectile interaction is obtained.

After solving numerically the radial wave function for the interaction potential, the transition amplitude in the peaking approximation reads:

$$
\begin{aligned}
& b_{C P C D W}=-i A_{0} \int_{0}^{\infty} d t^{\prime} e^{i\left(E-E_{0}\right) t^{\prime}-\frac{\Gamma}{2} t^{\prime}} t^{\prime i \frac{Z_{p}}{v_{P}}} \\
& \times\left(\sum_{l=0}^{\infty} R_{l}\left(\left|\mathbf{r}-\mathbf{v}_{P} t^{\prime}\right|\right) P_{l}(\cos (\theta)) \frac{1}{v^{\prime 1 / 2} e^{i \mathbf{v}^{\prime} \cdot\left(\mathbf{r}-\mathbf{v}_{\mathbf{p}} \mathbf{t}^{\prime}\right)}}\right)^{*} \\
& \approx-i A_{0} \sum_{l=0}^{\infty} \int_{0}^{\infty} d t^{\prime} e^{i\left(E^{\prime}-E_{0}-v_{P}^{2} / 2\right) t^{\prime}-\frac{\Gamma}{2} t^{\prime} t^{\prime i \frac{z_{p}}{v_{P}}} v^{\prime-1 / 2}} \\
& \times R_{l}\left(v_{P} t^{\prime}\right) P_{l}(\cos (\theta))
\end{aligned}
$$

where $E^{\prime}$ is the energy of the electron and $\theta$ is the polar angle in the projectile frame.

\section{RESULTS}

We now show and compare the results obtained by means of the three above mentioned models for the autoionization

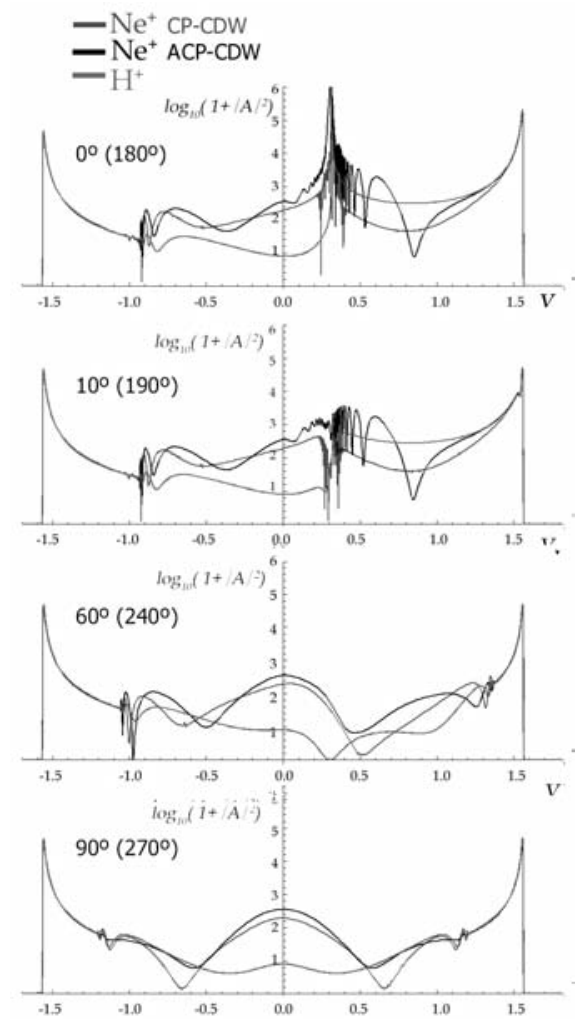

FIG. 5: $\mathrm{Ne}^{+}$impact velocity profile of $\log _{10}\left(1+|b|^{2}\right)$ for selected cuts of the velocity space: a) $\theta_{\text {emitter }}=0^{\circ}\left(180^{\circ}\right)$, b) $\theta_{\text {emitter }}=$ $10^{\circ}\left(190^{\circ}\right)$, c) $\theta_{\text {emitter }}=60^{\circ}\left(240^{\circ}\right)$, d) $\theta_{\text {emitter }}=90^{\circ}\left(270^{\circ}\right)$.

of He following the double excitation to the $2 s^{2}\left({ }^{1} S\right)$ state. For the photon impact case, the expected profie for the electronic spectrum consists in a Lorentzian centered around the resonant emission velocity $\widetilde{v}_{0}=1.5646$ a.u. with full width at half maximum $\Gamma=0.00507$ a.u. The inverse of the $\Gamma$-value is related to the lifetime of the autoionizing state. Although theoretical studies [9] have predicted that the presence of the receding projectile could modify the lifetime of the autoionizing state, we have employed in our calculations the constant photoionization value above mentioned.

In Figures 1 and 2 we show contour plots of the electronic distribution in momentum space for a projectile velocity of 0.316 a.u. which corresponds to $10 \mathrm{keV} / \mathrm{amu}$, an impact energy where many experiments have been performed $[6,10]$. We consider clothed projectiles with asymptotic charge +1 and varying nuclear charges. We first describe some common features related to this representation. i) The autoionization ring corresponding to $v_{e}=1.5646$ a.u. that represents the autoionization peak of the emitted electron spectrum and that would represent the sole expected feature for the photon impact case. ii) For the ion impact case, in addition to the autoionization ring (which would represent electrons not strongly deflected by the projectile) another structure is clearly visible: the binary ring centered in the projectile velocity value $(0.316$ a.u.) with a radius of 1.2486 a.u.. This structure is a typical signature of the postcollision interaction of electrons which pass close to the projectile and are strongly deflected. iii) The Coulomb focusing peak is located in the forward direction over the autoionization ring but in this rep- 
resentation turns hardly visible. In Figure 1 we compare the results obtained by means of the ACP-CDW and CP-CDW models for $\mathrm{H}^{+}$and $\mathrm{He}^{+}$impact. We observe that the main differences among these two models are obtained in the inner part of the binary ring. These are consequence of the different degree of accuracy in the representation of the emitted electron-projectile interaction. As mentioned above, the ACP-CDW model is not expected to be accurate at small $v^{\prime}$ values and in this case this limitation traduces in a much more involved oscillatory structure. The CP-CDW model, on the other hand, shows a smooth variation of the ditch-like structure that runs through the inner part of the binary ring as the projectile nuclear charge is increased up to +10 . For a better evaluation of these features, in Figs. 3-5 we show selected cuts of the electronic distributions in momentum space. In all cases the Coulomb focusing peak turns now visible in the forward direction for the $0^{\circ}$ cut. We note that all the models provide essentially the same results outside the binary ring. In all cases, the ACP-CDW and CP-CDW models predict an enhancement of the electronic density in the region where electrons backscattered by the projectile are expected. It is this enhancement and the oscillatory nature observed for the different cuts what has been interpreted in this context in terms of rainbow scattering [16]. For emission angles $<10^{\circ}$ case, the CP-CDW model also shows some oscillatory nature near $v^{\prime} \approx 0$ but in this case is related to the numerical discretization of $v^{\prime}$-values used. To avoid those oscillations a denser grid in $v^{\prime}$ must be considered largely increasing in this way the computational cost.

\section{CONCLUSIONS}

In this work, we have considered the autoionization of $\mathrm{He}$ induced by partially stripped ion impact. A CDW-like model has been proposed in order to obtain a quantum mechanical description of the autoionization process induced by clothed ion impact. The present model considers for the emitted electron-receding projectile a continuum wave function solution of a model central potential. For such a task we have used the parametrization provided by Garvey et al through which a large range of ionic species may be easily considered. The present model then gives a more precise description of the emitted electron-partially stripped projectile interaction at all times providing an ideal bench test for the applicability of the simpler analytical models here recalled.

Compared to the Barrachina-Macek model we observe that in qualitative terms the ACP-CDW and CP-CDW models provide similar results. A second inspection indicates that differences are obtained in the electronic spectra among these two models. Despite the fact the ACP-CDW model includes the information of the projectile-electron interaction through the non-Coulombic phase shifts, it seems clear that the interference between the two terms conforming Eq.(8) give rise to large oscillations nearby the $v^{\prime} \approx 0$ region. In this sense, the CP-CDW model then turns a more effective model to treat these processes and should be expected to be much more reliable. More experimental data for these type of collision processes would be welcome to help further improve the existing theoretical models.

\section{ACKNOWLEDGMENTS}

This work has been supported by PGI 24/F049 (UNS), PICT-2007-00887 of the ANPCyT and PIP 112-20080102760 of CONICET (Argentina).
[1] P. Auger, Jour. Radium 6, 205 (1925).

[2] R. B. Barker, H. W. Berry, Phys. Rev. 151, 14 (1966).

[3] A. Z. Devdariani, V. N. Ostrovskii, Yu. N. Sebayakin, Sov. Phys. JETP 46, 215 (1977).

[4] P. van der Straten, R. Morgenstern, J. Phys. B: At. Mol. Opt. Phys. 19, 1361 (1986).

[5] R. O. Barrachina, J. H. Macek, J. Phys. B: At. Mol. Opt. Phys. 22, 2151 (1989).

[6] J. K. Swenson, C. C. Havener, N. Stolterfoht, K. Sommer, F. W. Meyer, Phys. Rev. Lett. 63, 35 (1989).

[7] J. E. Miraglia, J. Macek, Phys. Rev. A 42, 3971 (1990).

[8] I. L. Cordrey, J. H. Macek, Phys. Rev. A 48, 1264 (1993).

[9] S. Otranto, C. R. Garibotti, F. D. Colavecchia, G. Gasaneo, Phys. Rev. A 63, 022713 (2001).

[10] J. K. Swenson, J. Burgdörfer, F. W. Meyer, C. C. Havener, D. C. Gregory, N. Stolterfoht, Phys. Rev. Lett. 66, 417 (1991).

[11] I. Samengo, R. G. Pregliasco, R. O. Barrachina, J. Phys. B: At. Mol. Opt. Phys. 32, 1971 (1999).

[12] Sh. D. Kunikeev, V. S. Senashenko, Sov. Phys. JETP 82, 839 (1996).

[13] C. O. Reinhold, D. R. Schultz, R. E. Olson, C. Kelbch, R. Koch and H. Schmidt-Böcking, Phys. Rev. Lett. 66, 1842 (1991).

[14] D. R. Schultz and R. E. Olson, J. Phys. B: At. Mol. Opt. Phys. 24, 3409 (1991).

[15] K. W. Ford and J. A. Wheeler, Ann. Phys. (N.Y.) 7, 259 (1959).

[16] S. Otranto and R. E. Olson, Phys. Rev. A 72, 022716 (2005).

[17] S. Otranto and G. Gasaneo, Phys. Scripta 70, 251 (2004).

[18] C. Cohen-Tannoudji, B. Diu, F. Laloë, Quantum Mechanics, Vol. II (John Wiley \& Sons, New York, 1977).

[19] I. S. Gradshteyn and I. M. Ryzhik, Tables of Integrals, Series and Products (London: Academic, 1965).

[20] Gy. Víkor, S. Ricz, B. Sulik, L. Tóth, A. Kövér, J. Végh, J. Phys. B: At. Mol. Opt. Phys. 29, L787 (1996).

[21] L. Schiff, Quantum Mechanics, (McGraw-Hill, New York, 1955)

[22] M. Abramowitz and A. Stegun, Handbook of Mathematical Functions (Washington, DC: NBS, 1964).

[23] R. H. Garvey, C. H. Jackman and A. E. S. Green, Phys. Rev. A 12, 1144 (1975).

[24] A. E. S. Green, D. L. Sellin and A. S. Zachor, Phys. Rev. 184, 1 (1969). 